\title{
ANÁLISIS EXPERIMENTAL DEL EFECTO DE CAMBIOS ESTRUCTURALES EN INVERNADEROS CON TECHO A DOS VERTIENTES
}

\author{
BouZO, C. A. ${ }^{1}$ \& LENSCAK, M. P. ${ }^{2}$
}

\begin{abstract}
RESUMEN
Fue realizado un análisis experimental de un invernadero a dos vertientes al cual se le realizaron modificaciones dimensionales y de diseño de las ventanas cenitales. Se compararon las mediciones internas del aire en su velocidad $\left(\mathrm{m} \mathrm{s}^{-1}\right)$, caudal $\left(\mathrm{m}^{3} \mathrm{~h}^{-1}\right)$, temperatura $\left({ }^{\circ} \mathrm{C}\right)$ y déficit de presión de vapor, DPV $(\mathrm{kPa})$ entre invernaderos denominados aquí "Capilla Modificado Tradicional" (CMT) y "Capilla Modificado Alternativo" (CMA). El trabajo fue realizado en Goya, Corrientes (29 02'S, $59^{\circ} 10^{\prime} \mathrm{W}$ ) durante los meses de febrero, marzo y noviembre del año 2016. Se observó una mejor condición micro-meteorológica en el invernadero CMA, al presentar menor temperatura interna diurna, mayores velocidades, caudal del aire y renovación horaria. Se discuten los resultados basados en las diferencias dimensionales y de diseño de los invernaderos teniendo en cuenta los resultados de otros trabajos de investigación.
\end{abstract}

Palabras claves: invernadero, estructura, micrometeorología, temperatura, renovación horaria.

\begin{abstract}
Experimental analysis of the effect of structural changes in greenhouses with a double-slope roof.

An experimental analysis of a two-slope greenhouse was carried out, to which dimensional modifications and design of the roof windows were made. Were compared the wind speed $\left(\mathrm{m} \mathrm{s}^{-1}\right)$, air flow rate $\left(\mathrm{m}^{3} \mathrm{~h}^{-1}\right)$, air temperature $\left({ }^{\circ} \mathrm{C}\right)$ and vapor pressure deficit $(\mathrm{kPa})$ in two greenhouses ca-

\footnotetext{
1- Facultad de Ciencias Agrarias, Universidad Nacional del Litoral. Laboratorio de Investigaciones en Fisiología y Biología Molecular Vegetal (LiFiBVe), Kreder 2805. (3080). Esperanza, provincia de Santa Fe. Email: cbouzo1160@gmail.com

2.- Instituto de Investigación y Desarrollo Tecnológico para la Agricultura Familiar Región Pampeana. Villa Elisa, La Plata Buenos Aires.

Manuscrito recibido el 15 de junio de 2018 y aceptado para su publicación el 15 de agosto de 2018
} 


\section{A. Bouzo et al.}

lled here CMT and CMA. The work was developed in Goya, Corrientes, Argentina (29 $02^{\prime} \mathrm{S}, 59^{\circ}$ $10^{\prime} \mathrm{W}$ ) in the year 2016 in february, march and november months. A better micro-meteorological condition was observed in the CMA greenhouse, since it had a lower daytime internal temperature, higher wind speed, air flow and consequently a greater ventilation-rate. The results are discussed based on the dimensional and design differences of the greenhouses taking into account the results of other research works.

Key words: greenhouse, structure, micrometeorology, temperature, ventilation-rate.

\section{INTRODUCCIÓN}

La construcción de invernaderos en Argentina se realizó principalmente imitando algunos tipos clásicos a nivel mundial, o bien como adaptaciones de estructuras utilizadas para otros fines como fue el caso de Corrientes, construyéndose los primeros invernaderos en la década de 1980 (9). Las primeras estructuras de invernaderos que se construyeron en esta provincia, surgieron de la adaptación de las estructuras denominadas "tendaleros" para el secado de hojas de tabaco (9). Posteriormente, fue durante la década siguiente la región de mayor concentración de invernaderos del país, ubicados en su mayoría en la costa del río Paraná, en las localidades de Bella Vista, Lavalle y Goya (19). En la actualidad, entre los tipos de invernaderos más utilizados se encuentran el denominado "rancho" o "capilla", que genéricamente se designa "a dos aguas" o también en otros países "a dos vertientes" (25). Sin embargo, el tipo de invernadero más difundido es el 'a dos aguas modificado', comúnmente denominado como "chileno", el cual consta de una estructura a dos aguas con ventilación cenital. Un tercer tipo que se encuentra actualmente en expansión es el metálico de techo curvo (21).

Para la producción hortícola en Argentina, la cual está orientada al mercado interno, la utilización de invernaderos siempre implica la obtención de un compromiso entre la sofisticación técnica de estas estructuras y el retorno económico que significa su adopción (24). Por tal motivo, los invernaderos artesanales con postes y maderos, cumplen su función de ejercer un forzado, aunque adolecen de fallas técnicas y de diseño que impiden la obtención de una mayor productividad de los cultivos durante el año (18).

A pesar de haber sido la provincia de Corrientes una región pionera en la producción hortícola mediante forzado en el país, actualmente se encuentra en discusión el tipo de invernadero más apropiado para la producción de tomate y pimiento, en consideración de los problemas originados durante los meses de mayor temperatura, comprendidos desde la primavera hasta el otoño, siendo particularmente muy dificultoso el cultivo en el período estival (19). Es así que con la tecnología actualmente empleada, consistente en la utilización de trasplante mediante genotipos de alto potencial de rendimiento, la temperatura excesivamente alta en el interior de los invernaderos resulta uno de los principales factores limitantes.

La altura cenital media de los invernaderos usados en la región es en general baja, si se tiene en cuenta su efecto sobre el balance energético durante los meses más cálidos. 
Estas dimensiones (altura lateral media de $2,0 \mathrm{~m} \pm 0,2 \mathrm{~m}$ y la cenital de 3,0 $\mathrm{m} \pm 0,4 \mathrm{~m}$ ) provocan un gran incremento de la temperatura a la altura del cultivo, agravándose el problema cuando aumenta el número de invernaderos acoplados lateralmente debido a la reducción proporcional de la ventilación lateral (4). El objetivo de este trabajo fue realizar un análisis experimental de la micro meteorología de dos invernaderos con techo a dos vertientes con ventilación cenital.

\section{MATERIALES Y MÉTODOS}

El trabajo consistió en comparar las condiciones micro-meteorológicas de un invernadero típico de la provincia de Corrientes, denominado aquí como "capilla modificado tradicional" (CMT) y un diseño alternativo denominado "capilla modificado alternativo" (CMA). Los dos tipos de invernaderos fueron construidos al mismo tiempo, en una empresa situada en la localidad de Goya, Corrientes $\left(29^{\circ}\right.$ $\left.02^{\prime} \mathrm{S}, 59^{\circ} 10^{\prime} \mathrm{W}\right)$. Los invernaderos CMT y CMA fueron separados por una calle interna y en cada caso formando parte de diferentes módulos acoplados lateralmente.

Los invernaderos CMT fueron acoplados en dos grupos de 5 invernaderos cada uno acoplados lateralmente y orientados de norte a sur. Cada grupo a su vez se encontraba separado por un pasillo de $6,00 \mathrm{~m}$ en sentido trasversal cubierto con polietileno para el tránsito de vehículos durante la cosecha.

Estos invernaderos fueron diseñados con una ventana cenital en toda su extensión longitudinal y una altura principal de 3,85 $\mathrm{m}$ y de 2,65 $\mathrm{m}$ de altura en los laterales. El ancho de cada invernadero fue de 7,10 $\mathrm{m} \mathrm{y}$ el largo de 50,00 m (Fig. 1). Las ventanas eran cenitales, frontales y laterales. En las cenitales la altura era de $0,15 \mathrm{~m}$ y $50 \mathrm{~m}$ de largo. La altura y longitud de las ventanas frontales era de $1,50 \mathrm{~m}$ de altura y $35,50 \mathrm{~m}$, respectivamente; representando un total de $53 \mathrm{~m}^{2}$ por cada ventana. A su vez, la altura y longitud de las ventanas laterales era de 1,50 $\mathrm{m}$ y $50,00 \mathrm{~m}$, respectivamente; lo que representó un total de $75 \mathrm{~m}^{2}$ cada una (Fig. 1).
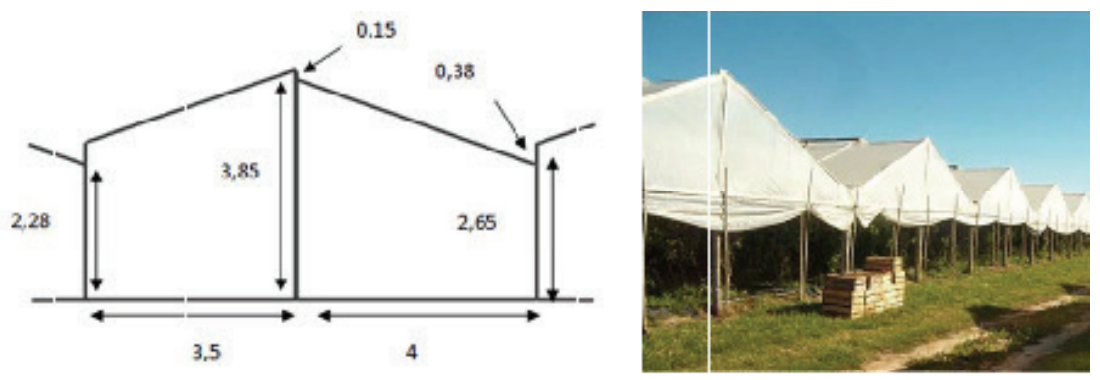

Figura 1: (izq.) Corte transversal y dimensiones ( $m$ ) (a) del invernadero con dos vertientes, denominado en este trabajo "Capilla Modificado Tradicional" (CMT); (der.) imagen en perspectiva de los invernaderos. 


\section{A. Bouzo et al.}

Los invernaderos CMA fueron también acoplados en dos grupos de 5 invernaderos cada uno orientados de norte a sur. Al igual que en el invernadero CMT cada grupo se encontraba separado por un pasillo en sentido trasversal cubierto con polietileno. Estos invernaderos se caracterizaron por poseer una mayor altura que el CMT, siendo la altura máxima de 4,30 m y 3,10 m de altura lateral. El ancho de cada invernadero era de 7,30 m y su longitud de 50,00 m (Fig. 2). Cada invernadero poseía una ventana cenital de $0,40 \mathrm{~m}$ de altura y $50,00 \mathrm{~m}$ de longitud. Las dimensiones de las ventanas frontales eran de $1,50 \mathrm{~m}$ de altura y $36,00 \mathrm{~m}$ de longitud representando una superficie total de $54 \mathrm{~m}^{2}$. Las ventanas laterales tenían 1,60 m de altura y $50,00 \mathrm{~m}$ de longitud totalizando una superficie de ventana de $80 \mathrm{~m}^{2}$. También existía una ventana lateral superior de 0,40 $\mathrm{m}$ de alto y 50,00 $\mathrm{m}$ de largo en la parte superior de las canaletas de desagüe, totalizando una superficie de $20 \mathrm{~m}^{2}$ cada una (Fig. 2).

$\mathrm{El}$ experimento fue dividido en tres fases experimentales, comprendiendo cada una de ellas períodos normalmente caracterizados por altas temperatura del aire durante el año 2016. El primer experimento se realizó en el mes de febrero, el segundo experimento estuvo comprendido entre finales de febrero e inicio del mes de marzo, y finalmente, el tercero se realizó en el mes de noviembre. En los dos primeros experimentos los invernaderos se encontraban sin cultivo, y en el último experimento se realizó con un cultivo de tomate cuyo índice de área foliar medio al momento de las mediciones era de $4 \mathrm{~m}^{2} \mathrm{~m}^{-2}$, y una altura de conducción de 2 $\mathrm{m}$. Para comparar las condiciones micro meteorológicas interiores de los invernaderos fueron medidas la humedad relativa $(\%)$, la temperatura $\left({ }^{\circ} \mathrm{C}\right)$, la velocidad $\left(\mathrm{m} \mathrm{s}^{-1}\right)$ y el caudal de aire $\left(\mathrm{m}^{3} \mathrm{~h}^{-1}\right)$. Además, en el tercer experimento el balance energético del cultivo fue estimado indirectamente mediante la medición de la temperatura de las plantas $\left({ }^{\circ} \mathrm{C}\right)$. La temperatura del aire fue medida mediante 12 termómetros distribuidos en cada invernadero (CMT y CMA) en el módulo central, los que fueron instalados a 2,00 m de altura, distribuidos en doce puntos de observación equivalentes en su distribución en los dos tipos de invernaderos. La velocidad y caudal de aire en el interior del invernadero fue medido con un anemómetro térmico Testo 405, y la humedad relativa con 12
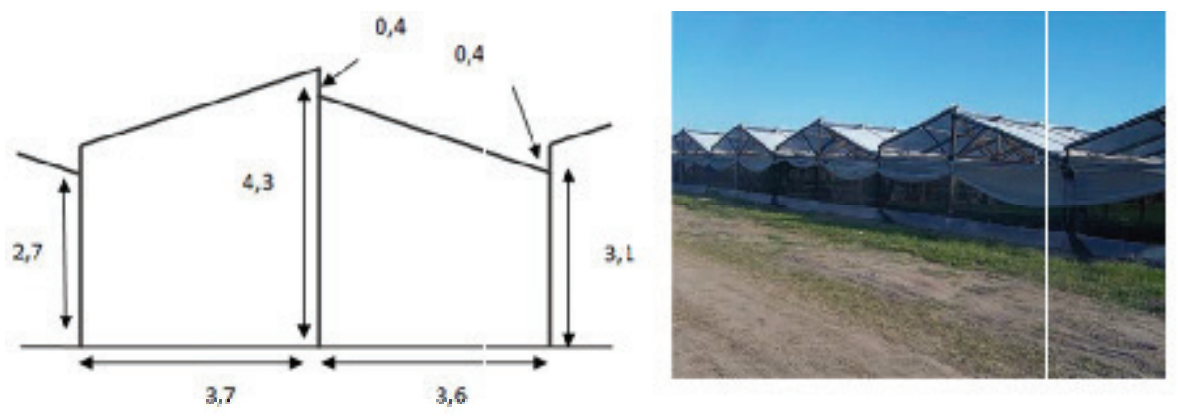

Figura 2. (izq.) Corte transversal y dimensiones ( $m$ ) del invernadero con dos vertientes denominado en este trabajo "Capilla Modificado Alternativo"(CMA); (der.) imagen en perspectiva de los invernaderos. 
psicrómetros de bulbo húmedo. En el tercer experimento se midió la temperatura de las plantas mediante un termómetro infrarrojo Crop TRAK Mini IR Thermometer. En todos los casos, las observaciones fueron directas, y efectuadas en durante 7 días consecutivos, a las 8.00, 13.00 y 17.00 hs.

Las condiciones meteorológicas externas fueron medidas mediante una estación meteorológica automática $\mathrm{WH}$ $1081\left(-40^{\circ} \mathrm{C} /+65^{\circ} \mathrm{C}\right)$ situada a $25 \mathrm{~m} \mathrm{de}$ los invernaderos estudiados. El diseño estadístico fue multifactorial y los re- sultados obtenidos en los tres experimentos fueron analizados mediante el test de Tukey con un 95\% de confianza.

\section{RESULTADOS}

La velocidad del aire medida a $2 \mathrm{~m}$ de altura resultó mayor en el invernadero CMA para la hora 13.00 y 17.00 en los experimentos 1 y 3 (Figs. 3 a y 5 a, respectivamente). En el experimento 2 también fue mayor
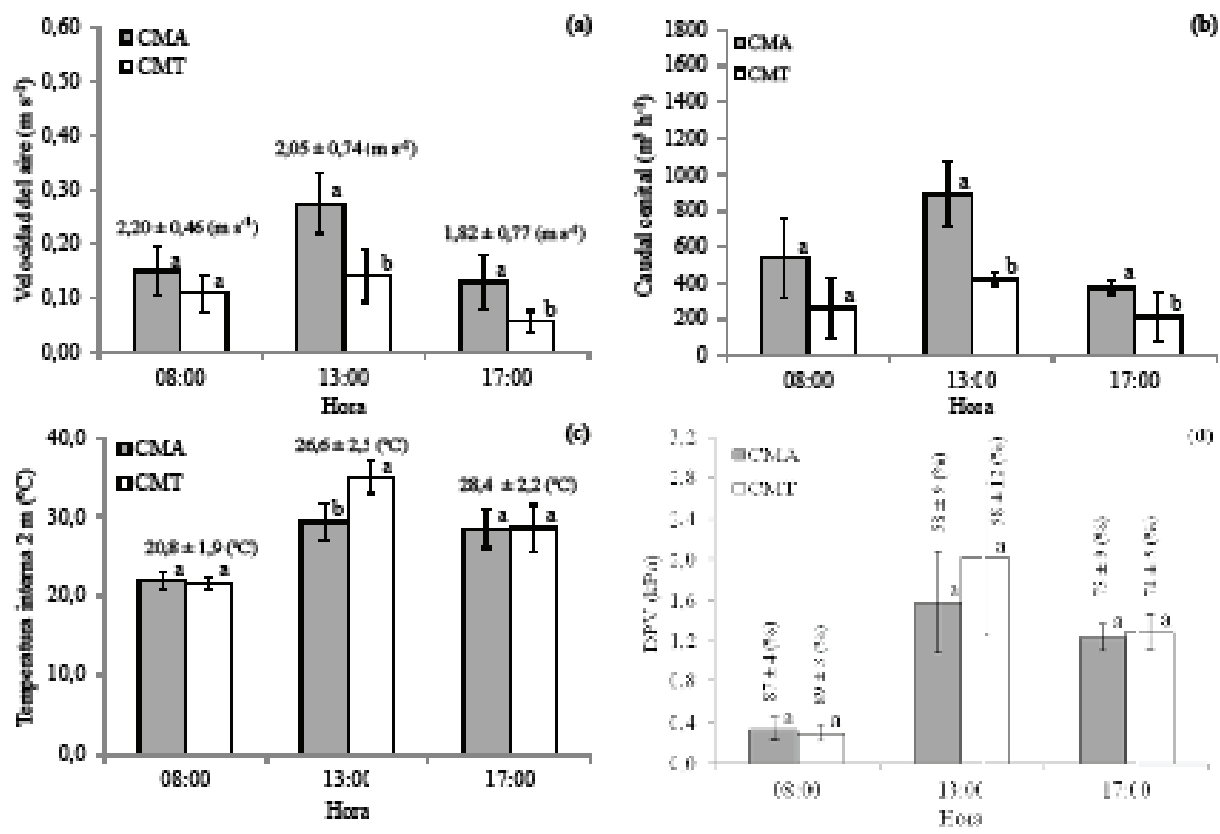

Figura 3. Efecto de los invernaderos 'Capilla Modificado Alternativo"(CMA) y "Capilla Modificado Tradicional" (CMT) sin cultivo sobre la velocidad del aire $\left(m \mathrm{~s}^{-1}\right)$, caudal en las ventanas cenitales $\left(\mathrm{m}^{3} \mathrm{~h}^{-1}\right)$, temperatura interna $\left({ }^{\circ} \mathrm{C}\right)$ y déficit de presión de vapor, $\mathrm{DPV}(\mathrm{kPa})$ en la fase experimental 1. Las líneas en cada barra representan el desvio estándar. Los valores sobre cada barra en las figuras (a) y (c), representan la media y el desvío estándar de cada factor en el ambiente externo a los invernaderos, y en la figura (d) la humedad relativa externa. Letras diferentes en cada hora representan diferencias estadisticas según el test de Tukey $(P \leq 0,05)$. 
pero solamente a la hora 13.00 (Fig. 4a). Incluso en el experimento 3 , se obtuvieron diferencias estadísticas $(\mathrm{P} \leq 0,05)$ en los tres horarios (Fig. 5a). Por lo tanto, en las tres fases experimentales a la hora 13.00 se midieron velocidades del aire mayores en el invernadero CMA que en CMT. El caudal cenital, que permite estimar el volumen de aire que sale de las ventanas superiores por unidad horaria, fue mayor en el invernadero CMA también a la hora 13.00 pero sólo en el experimento 1 (Fig. 3b) y 2 (Fig. 4b). En la fase experimental 3 no se observaron diferencias estadísticas $(\mathrm{P} \leq 0,05)$ entre los dos tipos de invernaderos (Fig. 5b). En este experimento, aunque los valores medios de caudal fueron mayores en el invernadero CMA, las dispersiones medidas a través del desvío estándar fueron también mayores. La temperatura interna medida a $2 \mathrm{~m}$ de altura presentó diferencias también entre las fases experimentales. Aunque en la fase 2 no se observaron diferencias entre los dos tipos de invernaderos (Fig. 4c), en las fases 1 y 3 se observaron diferencias a la hora 13.00 con mayores valores de temperatura
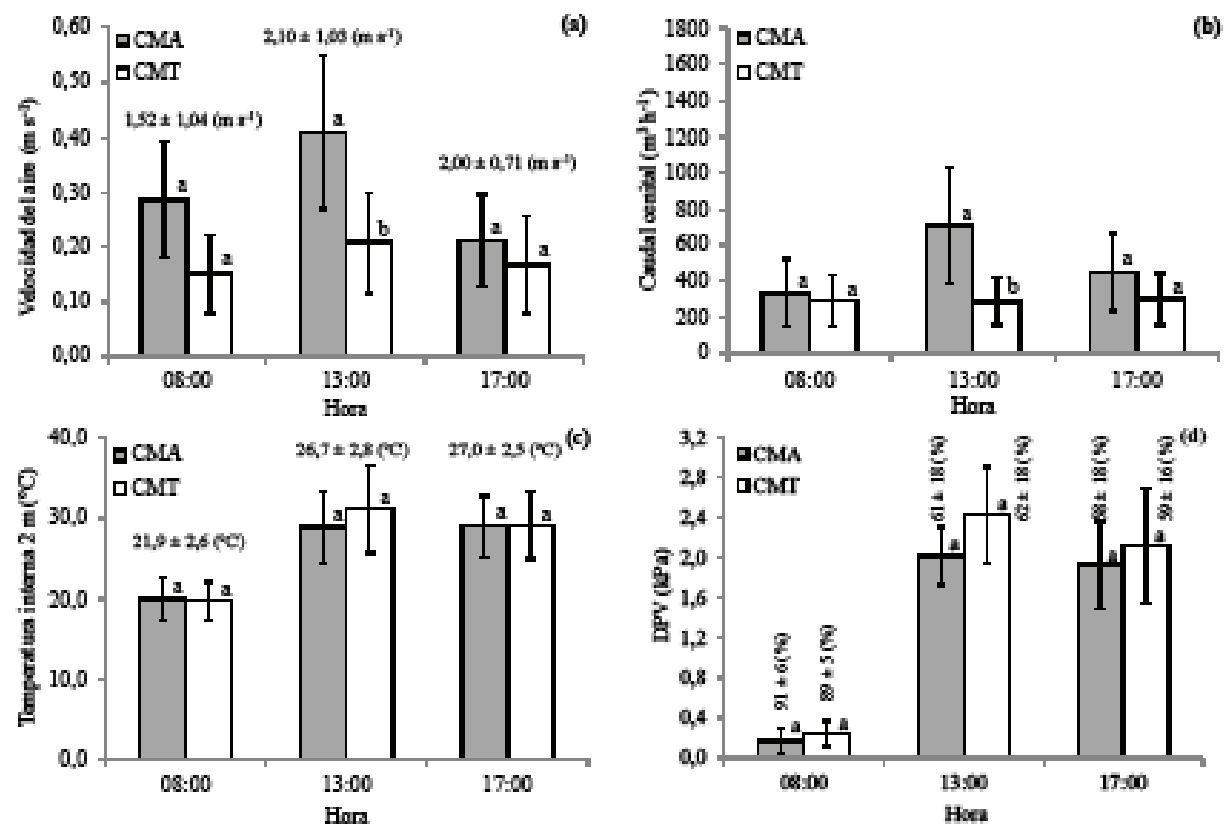

Figura 4. Efecto de los invernaderos 'Capilla Modificado Alternativo" (CMA) y "Capilla Modificado Tradicional" (CMT) sin cultivo sobre la velocidad del aire $\left(m \mathrm{~s}^{-1}\right)$, caudal en las ventanas cenitales $\left(m^{3} h^{-1}\right)$, temperatura interna $\left({ }^{\circ} \mathrm{C}\right)$ y déficit de presión de vapor, $D P V(\mathrm{kPa})$ en la fase experimental 2. Las líneas en cada barra representan el desvio estándar. Los valores sobre cada barra en las figuras (a) y (c), representan la media y el desvío estándar de cada factor en el ambiente externo a los invernaderos, y en la figura (d) la humedad relativa externa. Letras diferentes en cada hora representan diferencias estadisticas según el test de Tukey $(P \leq 0,05)$. 
en el invernadero CMT (Fig. 3c y 5c). En la fase 1 a la hora $13.00 \mathrm{y}$ en el invernadero CMT, la temperatura fue en promedio para todo el período de medición de $5,6^{\circ} \mathrm{C}$ mayor (Fig. 3c) y en la fase 3 de $6,2^{\circ} \mathrm{C}$ (Fig. 5c).

Las mediciones realizadas en la humedad relativa expresada como déficit de presión de vapor del aire no presentaron diferencias estadísticas entre los dos tipos de invernaderos en las tres fases experimentales
(Figs. 3d, 4d y 5d). Finalmente, en la fase 3, la temperatura foliar en el cultivo permitió determinar la existencia de diferencias estadísticas $(\mathrm{P} \leq 0,05)$ entre los dos tipos de invernaderos a la hora 13.00 (Fig. 6), habiéndose observado en el invernadero CMT una temperatura de las hojas de $4,3^{\circ} \mathrm{C}$ mayor en comparación con el invernadero CMA.
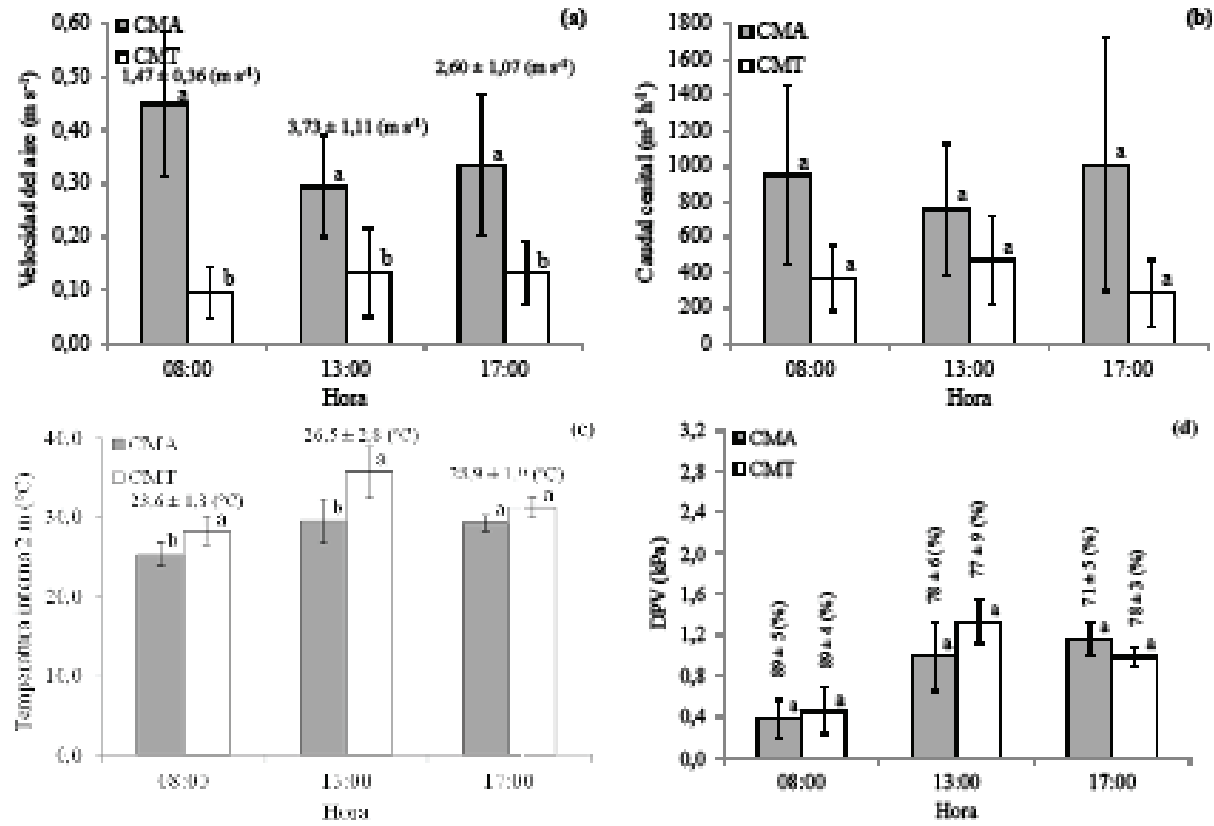

Figura 5. Efecto de los invernaderos 'Capilla Modificado Alternativo"(CMA) y "Capilla Modifcado Tradicional" (CMT) con un cultivo de tomate sobre la velocidad del aire ( $\left.m \mathrm{~s}^{-1}\right)$, caudal en las ventanas cenitales $\left(\mathrm{m}^{3} \mathrm{~h}^{-1}\right)$, temperatura interna $\left({ }^{\circ} \mathrm{C}\right)$ y déficit de presión de vapor, $D P V(\mathrm{kPa})$ en la fase experimental 3. Las líneas en cada barra representan el desvio estándar. Los valores sobre cada barra en las figuras (a) y (c), representan la media y el desvío estándar de cada factor en el ambiente externo a los invernaderos, y en la figura (d) la humedad relativa externa. Letras diferentes en cada hora representan diferencias estadisticas según el test de Tukey $(P \leq 0,05)$. 


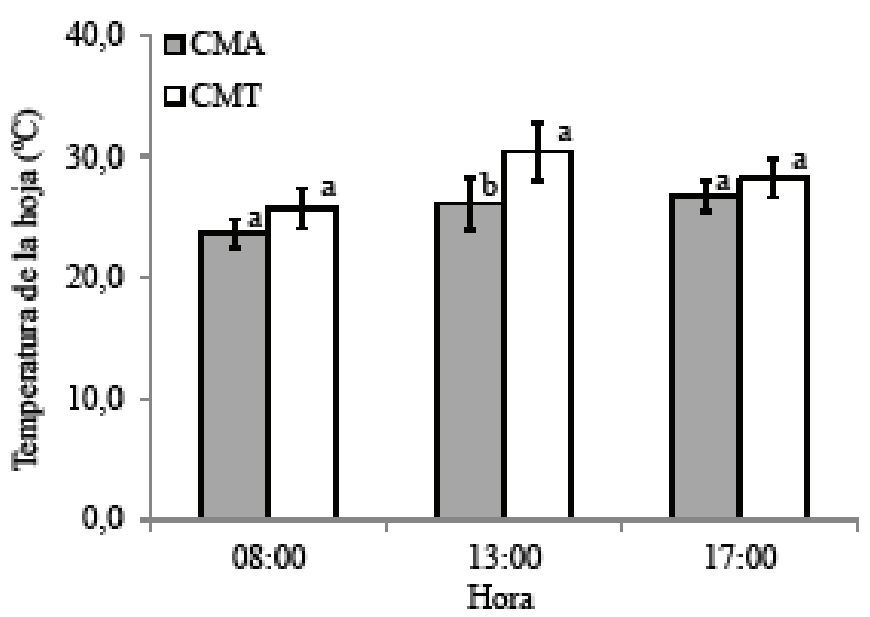

Figura 6. Efecto de los invernaderos 'Capilla Modificado Alternativo"(CMA) y "Capilla Modificado Tradicional" (CMT) en la fase experimental 3 sobre la temperatura de las plantas de tomate. Las lineas en cada barra representan el desvio estándar. Letras diferentes en cada hora representan diferencias estadísticas según el test de Tukey $(P \leq 0,05)$.

\section{DISCUSIÓN}

Es usual que la ventilación pasiva mediante ventanas laterales y cenitales resulte insuficiente para provocar una adecuada renovación del aire interior de los invernaderos, por lo que las velocidades medidas entre el exterior e interior disminuyen considerablemente (3). En los experimentos realizados se pudo observar que tomando en conjunto todas la mediciones realizadas en los tres fases experimentales, la velocidad del aire externa resultó entre 5 a 14 veces mayor que las medidas en el interior de los invernaderos (Figs. 3a, 4a y 5a). Sin embargo, de lograrse una adecuada resolución de los sistemas de ventilación natural, se pueden mejorar los procesos de transferencia de energía entre el interior y exterior del invernadero (24). En este trabajo, las diferencia medidas entre los dos invernaderos en las velocidades del viento a la hora 13.00 , coinciden aproximadamente con el momento en que ocurre la temperatura máxima diaria, representan una ventaja para el caso del invernadero CMA con respecto a CMT (Figs 3a, 4a y 5a). Al relacionar las superficies de ventanas laterales con respecto a la superficie de suelo cubierto, los dos tipos de invernaderos tuvieron un valor de $11 \%$. La diferencia no obstante estuvo dada en la superficie de ventanas cenitales, ya que en el invernadero CMA fue de $11 \%$ y en el CMT de 3\%. Por lo que relacionando ahora las superficies totales de ventanas con respecto a la superficie de suelo, en el invernadero CMA fue de $22 \%$ y en el CMT fue de $13 \%$. De esta manera, la proporción de ventanas del invernadero CMA la sitúa en una mejor condición para la ventilación si se considera que algunos autores determinaron como adecuado un rango de 15 a $25 \%$ 
(14), 25\% (34), 10 a $30 \%(31,32)$. Otros autores $(22,10)$ recomiendan que este valor no sea inferior a $20 \%$. Por lo tanto, la proporción de ventanas superior en el invernadero CMA con respecto a CMT explicaría la mayor velocidad del viento medida en el primero. Además, el valor de $22 \%$ para el invernadero CMA resultó superior al promedio de los tipos de invernaderos dominantes en la provincia de Corrientes (19). Aunque la mayor velocidad del aire fue medida en CMA en las tres fases experimentales a la hora 13.00 (Figs. 3a, 4a y 5a), es conveniente analizar las proporciones de ventanas laterales y cenitales. Para el caso de las ventanas cenitales, la proporción con respecto a la superficie del suelo para el invernadero CMA lo sitúa muy por encima a la del invernadero CMT. Aunque no existen precisiones en cuanto al valor que debiera tener la proporción de ventanas cenitales con respecto a la superficie de suelo cubierto, se considera aceptable si se encuentra en un rango de 5 a $10 \%$ (24). Por lo tanto, el invernadero CMA tuvo una proporción de ventanas cenitales mayores al $10 \%$, y por otra parte resultó superior a la media medida en invernaderos de este tipo en la provincia de Corrientes (19). La ventilación cenital incrementa su importancia con la disminución de la velocidad del viento externa, por lo que en determinadas situaciones de muy baja velocidad de viento, la renovación del aire ocurre principalmente por efecto convectivo o de flotación (2). El efecto de flotación sobre la ventilación de un invernadero debe ser considerado cuando la velocidad del viento es inferior a 1,8 $\mathrm{m} \mathrm{s}^{-1}$ (26). Sin embargo, posteriormente se profundiza este criterio (15), estableciendo que la importancia relativa del efecto térmico por flotación y la fuerza provocada por el viento depende de la razón entre la velocidad del viento y la raíz cuadrada de la diferencia entre la temperatura interna y externa del invernadero. De acuerdo a esta relación, el efecto del viento domina sobre el de la flotación cuando esta relación es mayor a la unidad. En este trabajo, el cálculo realizado sobre los valores medios de velocidad del viento externa, temperaturas internas y externas a la hora 13.00, permite deducir que en el invernadero CMA en todos los casos fue más importante el efecto del viento, ya que el índice calculado fue de $1,25,1,41$ y 2,15 para las fases experimentales 1, 2 y 3, respectivamente. En el invernadero CMT estos índices fueron de $0,71,1,00$ y 1,22 para cada fase. Estos valores indicarían que con la combinación de entre superficies de ventanas laterales y cenitales fue más importante el efecto del viento en el invernadero CMA que en el invernadero CMT, y también el que explica la mayor velocidad de viento interna medida en CMA. Otra cuestión que aconsejaría un análisis más pormenorizado, es el efecto de la particular combinación de las ventanas cenitales en el invernadero CMA, al haberse encontrado distribuidas entre las situadas a la altura de las canaletas y las propias del extremo superior del techo.

La velocidad del viento medida a $2 \mathrm{~m}$ de altura en el interior de los invernaderos representa aproximadamente la mitad de la altura de los mismos. Al mismo tiempo, si se considerara a esta velocidad como un promedio del perfil vertical de los vectores de viento, calculando el volumen de los invernaderos se puede deducir las renovaciones horarias. Bajo estos supuestos, el número de renovaciones en el invernadero CMA a las 13.00 hs hubieran representado $20 \mathrm{~h}^{-1}, 29 \mathrm{~h}^{-1}$ y $21 \mathrm{~h}^{-1}$ para fases experimen- 
tales 1, 2 y 3, respectivamente. En tanto que para el invernadero CMT y para las respectivas fases, las renovaciones estima-

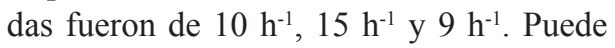
observase que en todos los casos, las renovaciones horarias en el invernadero CMA fueron casi el doble de las del invernadero CMT. A los fines de tener una comparación a partir de estos valores calculados de renovación del aire, debe considerarse que la necesidad de ventilación de los invernaderos pueden modificarse según los cultivos y estación de crecimiento, con valores que oscilarían entre $2 \mathrm{~h}^{-1}$ en invierno hasta $60 \mathrm{~h}^{-1}$ en verano (20). Para el caso de situaciones estaciones cálidas, al último valor presentado precedentemente, se agrega como recomendación general que son deseables renovaciones superiores a $40 \mathrm{~h}^{-1}$ (24) y que en particular sería de aplicación para invernaderos situados en Corrientes y en meses similares a los de este trabajo. Si se sigue este criterio entonces, tanto los invernaderos CMA como CMT son deficientes en renovación del aire, aunque la situación sería mucho más comprometida en CMT. Otro aspecto a considerar como favorable en el invernadero CMA con respecto al CMT es la diferencia en altura. En otro trabajo (4), hemos estudiado el efecto de la altura y demostramos que una mayor altura aumentó la inercia térmica, acelerando el efecto de flotación e incrementando las renovaciones de aire. Aunque el presente es un estudio general sobre el efecto de dos configuraciones de invernaderos sobre su micro meteorología, no puede desconocerse la necesidad de profundizar las investigaciones considerando además la dirección del viento y el efecto de modificar la disposición de las ventanas, dado que en climas cálidos se ha demostrado el beneficio de la ventilación a barlovento sobre las de sotavento (29). $\mathrm{Al}$ analizar los resultados obtenidos de la medición del caudal cenital se observó en primer lugar el efecto producido por la presencia del cultivo (Fig. 5b). En comparación con las fases experimentales 1 y 2 a la hora 13.00, en el experimento 3 la presencia del cultivo de tomate podría explicar la ausencia de diferencias en los valores medidos del caudal cenital $\left(\mathrm{m}^{3} \mathrm{~h}^{-1}\right)$, aun teniendo el invernadero CMA una proporción de 8\% mayor de ventanas cenitales con respecto a CMT. Es conocido a partir de estudios realizados en física de invernaderos, que la presencia de un cultivo produce fuertes perturbaciones en comparación con los invernaderos vacíos. Por ejemplo, de la comparación de situaciones con y sin cultivo se dedujo para el primer caso una atenuación en los perfiles de temperatura verticales y una mayor homogeneización, con una reducción de la turbulencia del aire (20). Por otra parte, y aunque fue demostrado el efecto combinado del gradiente de temperatura interior y el viento externo (33) la presencia del cultivo atenúa el efecto de este último factor ambiental, representando en casos de cultivos altos una fuerte resistencia al flujo de aire que ingresa al invernadero (28). Específicamente para el caso de un cultivo de tomate en total desarrollo, el mismo se comporta físicamente como un medio poroso, generando una caída de presión y disminuyendo la turbulencia del aire (5). En los últimos años un importante utilización de programas sustentados sobre la base de la dinámica computacional de fluidos (CFD) son utilizados para estudios de física de invernaderos, por lo que resultaría un importante avance su aplicación para validar los resultados experimentales obtenidos en este trabajo (13). 
Considerando ahora la temperatura interna medida, se destaca el hecho que en el invernadero CMA los valores nunca fueron mayores a los del invernadero CMT (Figs. $3 \mathrm{c}, 4 \mathrm{c}$ y $5 \mathrm{c}$ ). Para analizar estos resultados, en primer lugar debe tenerse en cuenta que la temperatura interna de un invernadero depende de varios factores propios del balance de energía y masa del mismo (7). Entre aquellos se encuentran la transmitancia del material de cobertura, la intensidad de la radiación solar, el intercambio de calor sensible y latente, el gradiente de presión de vapor y el coeficiente de transferencia por conducción de la cubierta (7). Una interpretación general de estos factores en la ecuación de balance permite observar que la mayoría son comunes a ambos tipos de invernaderos, a excepción del intercambio de calor sensible y latente, que en gran medida dependen de la renovación de aire del invernadero. No obstante, debe también tenerse en cuenta que la ventilación influye sobre el flujo de calor al suelo $(12,27)$, el que no fue medido en este trabajo. Sin embargo, podría afirmarse que las diferencias encontradas entre los dos tipos de invernaderos en la velocidad interna del aire (Figs. 3a, 4a y 5a) explicaría en gran parte las diferencias de temperaturas medidas. Por lo anterior, es evidente que para las condiciones climáticas de Corrientes deban coexistir los dos tipos de ventanas: lateral y cenital, siendo el invernadero CMA el que tuvo el mejor comportamiento. Son numerosos los antecedentes en donde se observó que el uso combinado de ambas incrementa la velocidad y disminuye la temperatura del aire interior, aunque también incrementa la heterogeneidad espacial del microclima del invernadero, en comparación con invernaderos ventilados sólo con ventanas laterales o cenitales (16).

Desde el punto de vista psicrométrico, la humedad relativa y el punto de rocío son los indicadores más ampliamente utilizados para expresar la cantidad de vapor de agua en el aire (17). No obstante existen amplias referencias también a favor del déficit de presión de vapor (DPV) utilizado en este trabajo como el indicador más apropiado para expresar la fuerza del transporte de agua desde la planta hacia el aire (35). Este fenómeno, el de la transpiración puede ser expresada como la suma del componente radiactivo, proporcional a la radiación absorbida por el cultivo y el advectivo, proporcional al déficit de saturación del aire (6, 23). La ausencia de diferencias estadísticas en el DPV del aire de los dos tipos de invernaderos estudiados (Fig. 5d) indicaría similares tasas transpiratoria del cultivo. Pero al haberse medido diferencias estadísticas en la temperatura de las plantas $(\mathrm{P} \leq 0,05)$ implica que las mismas deban haberse dado por otro factor, considerando que la temperatura es uno de las principales resultantes del balance de radiación y energía del cultivo (8). La diferencia de casi $4^{\circ} \mathrm{C}$ resultante del promedio de las mediciones a las 13.00 hs, indicaría una situación más favorable para el cultivo del invernadero CMA (Fig. 6). Este resultado y la ausencia de diferencias en el DPV del aire, indicarían entonces que las diferencias en la temperatura del cultivo se explicaría principalmente por la pérdida de calor conductivo-advectivo al ser menor la temperatura del aire en CMA y mayor su velocidad interna (Figs. 5a y 5c). Ambos factores influyen directamente en una mayor pérdida de calor al aumentar el gradiente térmico por un lado y disminuir la resistencia de la capa límite por el otro (11). Finalmente ambos términos, el DPV y la 
temperatura, son importantes ya que cuando el primero es moderado, posibilita una transpiración activa y un rendimiento alto en tomate (30). Por otra parte, con valores de temperatura de 10 a $30^{\circ} \mathrm{C}$ se observó una tasa de crecimiento de los frutos positivamente y linealmente relacionada a dicho incremento (1). Al respecto se destaca que en el invernadero CMT la temperatura del aire a las 13.00 hs fue de casi $36^{\circ} \mathrm{C}$, mientras que en CMA fue de $29,5^{\circ} \mathrm{C}$ (Fig. $5 \mathrm{c}$ ), resultando por lo tanto desde el punto de vista del crecimiento de los frutos más favorable la situación medida en el invernadero CMA.

\section{CONCLUSIONES}

En el invernadero CMA se obtuvo una mejor condición micro-meteorológica principalmente en el mediodía astronómico. A las 13 horas en el invernadero CMA fueron medidas una menor temperatura interna, mayor velocidad del aire, habiéndose deducido una mayor renovación horaria. El invernadero CMA presentó un mayor caudal cenital del aire, con excepción a las mediciones realizadas en el mes de noviembre. En este mismo mes, en este invernadero la temperatura del cultivo fue de casi $4^{\circ} \mathrm{C}$ menor en comparación con el CMT.

\section{AGRADECIMIENTOS}

Los autores expresan su más sincero agradecimiento a los Ing. Agrs. Gonzalo Nagel y Santiago Brest por los trabajos de campo, sin cuya colaboración no hubiera sido posible la realización de este trabajo.

\section{BIBLIOGRAFÍA}

1.- ADAMS, S.R.; COCKSHULL, K.E.; CAVE, R.J. 2001. Effect of temperature on the growth and development of tomato fruits. Annals of Botany 88:869-877.

2.- BOT, G. P. A.; VAN DE BRAAK, N. J. 1995. Physics of greenhouse climate. In: Bakker, J. C., Bot, G. P. A., Challa, H., van de Braak, N. J. (eds) Greenhouse climate control - an integrated approach. Wageningen: Wageningen Pers. 279 p.

3.- BOT, G.P.A. 1983. Greenhouse climate: from physical processes to a dynamic model. Ph.D. Dissertation, Agricultural University of Wageningen, The Netherlands, pp. 240.

4.- BOUCHET, E.R.; FREYRE, C.E.; BOUZO, C.A.; FAVARO, J.C. 2007. Efecto de las dimensiones de un invernadero sobre la temperatura interna en períodos cálidos. RCA 11(2):111-119.

5.- BOULARD, T.; KITTAS, C.; ROY, J.C.; WANG, S. 2002. Convective and ventilation transfers in greenhouses. Part 2: Determination of the distributed greenhouse climate. Biosystems Engineering 83(2):129-147.

6.- BOULARD, T.; WANG, S. 2002. Experimental and numerical studies on the heterogeneity of crop transpiration in plastic tunnel. Computers and Electronics in Agriculture, 34:173-190.

7.- BOUZO, C.A.; GARIGLIO, N.F.; PILATTI，R.A.; GRENON，D.A.; FAVARO, J.C.; BOUCHET, E.R.; FREYRE, C. 2006. 'InverSim': A simulation model for a greenhouse. Acta Hortic. 719:271-278.

8.- BOUZO, C.A.; NORERO SCHIAFFINO A.L.2015. Mathematical model for predicting the maximum water demand of crops. FAVE Ciencias Agrarias. Argentina 14(1):33-59.

9.- CASTRO, J.E. 2006. Producción hortícola de primicia en Corrientes. EEA INTA Bella Vista. Serie Técnica $N^{\circ}$ 18:16-22. 
10.- CONNELLAN G.J. 2002. Selection of greenhouse design and technology options for high temperature regions. Acta Hort. 578:113-117.

11.- FRICKE, W. 2017. Water transport and energy. Plant Cell Environ. 40(6):977-994.

12.- FUCHS, M.; DAYAN E.; SHMUEL D.; ZIPORI, I. 1997. Effects of ventilation on the energy balance of a greenhouse with bare soil. Agricultural and Forest Meteorology 86(3-4):273-282.

13.- HE, K.; CHEN, D.; SUN, L.; LIU, Z.; HUANG, Z. 2015. The effect of vent openings on the microclimate inside multi-span greenhouses during summer and winter seasons. Engineering Applications of Computational Fluid Mechanics 9(1):399-410.

14.- KACIRA, M.; SASE, S.; OKUSHIMA, L. 2004. Effects of side vents and span numbers on wind-induced natural ventilation of a gothic multi-span greenhouse. Japan Agricultural Research Quarterly, JARQ 38(4):227-233.

15.- KITTAS, C.; BOULARD, T.; PAPADAKIS, G. 1997. Natural ventilation of a greenhouse with ridge and side openings: Sensitivity to temperature and wind effects. Transactions of the ASAE 40(2):415-425. 16.- KITTAS, C.; KATSOULAS, N.; BARTZANAS, T.; MERMIER, M.; BOULARD, T. 2008. The impact of insect screens and ventilation openings on the greenhouse microclimate. Transactions of the ASABE 51(6):1-15.

17.- LAWRENCE, M.G. 2005. The relationship between relative humidity and the dewpoint temperature in moist air. A simple conversion and applications. Bulletin of the American Meteorological Society. February 225-233.

18.- LENSCAK, M.P.; BOUZO, C.A.; CÁCERES, S.; COLOMBO, M.H.; FERNÁNDEZ, H.; FERNÁNDEZ, R.; IGLESIAS, N.; MARTÍNEZ QUINTANA, O.; UVIEDO, R. 2004. Modelos de estructuras de invernaderos en la República Argentina.
En: Invernaderos: La experiencia Iberoamericana. Cyted. Programa Iberoamericano de Ciencia y Tecnología para el desarrollo. Editores: Acuña Caita, J. F.; Valera Martinez, D. y J. C. Avendaño. Almeria, España. p. 197. 19.- LENSCAK M.P.; MANSUTTI, J.J. 2009. Relevamiento y diagnóstico técnico de las estructuras de invernaderos de la provincia de Corrientes. INTA EEA Bella Vista. Serie Técnica $N^{\circ} 33.18$ p. 20.- LÓPEZ MARTÍNEZ, A. 2011. Contribución al conocimiento del microclima de los invernaderos mediterráneos mediante anemometría sónica y termografía. Tesis Doctoral. Universidad de Almería, España. 605 p. 21.- MANGO A.; DÍAZ S. 2010. El cultivo de pimiento bajo invernadero en Corrientes. Tesis de grado. Universidad del Salvador, Facultad de Agronomía. Gobernador Ingeniero Valentín Virasoro. 39 p.

22.- MONTERO, J.I.; ANTÓN, A.; KAMARUDDIN, R.; BAILEY, B.J. 2001. Analysis of thermally driven ventilation in tunnel greenhouses using sma11 scale models. Journal of Agricultural Engineering Research, 79:213-222.

23.- MONTERO, J.I. 2006. Evaporative cooling in greenhouses: effect on microclimate, water use efficiency and plant response. Acta Hort. 719, 373-383.

24.- MONTERO, J.I.; STANGHELLINI, C.; CASTILla, N. 2009. Greenhouse technology for sustainable production in mild winter climate areas: trends and needs. Acta Hortic. 807:33-44.

25.- ORTIZ CERECERES, J.; SÁNCHES DEL CASTILlO, F.; MENDOZA CASTILLO, M.C.; TORRES GARCÍA, A. 2009. Características deseables de plantas de pepino crecidas en invernadero e hidroponía en altas densidades de población. Revista Fitotecnia Mexicana 32(4):289-294. 
26.- PAPADAKIS, G.; MERMIER, M.; ME- 30.- TRIGUI， M.; BARRINGTON, S.F.; NESES, F.; BOULARD, T. 1996. Measurements and analysis of air exchange rates in a greenhouse with continuous roof and side openings. J. Agric. Eng. Res. 63(3): 219-228.

27.- PÉREZ-PARRA, J.; MONTERO, J.I.; BAEZA, E.; ANTÓN, A. 2001. Ventilación y refrigeración de invernaderos. En: López, J.C., Lorenzo, P., Castilla, N., Pérez-Parra, J., Montero, J.I., Baeza, E., Antón, A., Fernández, M.D., Baille, A.,González-Real, M. 2001. Incorporación de tecnología al invernadero mediterráneo. Caja Rural de Almería y Málaga (CAJAMAR). Almería, España. p. 49-58.

28.- RUIZ-GARCÍA, A.; LÓPEZ-CRUZ I.L.; ARTEAGA-RAMÍREZ， R.; RAMÍREZ-ARIAS, J.A. 2015. Tasas de ventilación natural de un invernadero del centro de México estimadas mediante balance de energía. Agrociencia 49:87-100. 29.- SASE, S. 2006. Air movement and climate uniformity in ventilated greenhouses. Acta Hort. 719:313-324. GAUTHIER, L. 1999. Effects of humidity on tomato (Lycopersicon esculentum cv. Truss) water uptake, yield, and dehumidification cost. Canadian Agricultural Engineering 41(3):135-140.

31.- VERHEYE, P.; VERLODT, H. 1990. Comparison of different systems for static ventilation of hemispheric plastic greenhouses. Acta Hort. 281:183-197. 32.- VON ZABELTITZ, C.H.R. 1992. Current state of technology and introduction to innovation in greenhouse horticulture. Acta Hort. 312:19-28.

33.- WANG, S.; DELTOUR, J. 1998. Theoretical study of natural ventilation flux in a single span greenhouse. Biotechnol. Agron. Soc. Environ. 2(4):256-263).

34.- ZABELTITZ, C. 1990. Greenhouse construction in function of better climate control. Acta Hort. 263:357-374. 35.- ZHANG, D.; DU, Q.; ZHANG, Z.; JIAO, X.; SONG, X.; LI, J. 2017. Vapour pressure deficit control in relation to water transport and water productivity in greenhouse tomato production during summer. 